\section{Patient-Physician E-Mail}

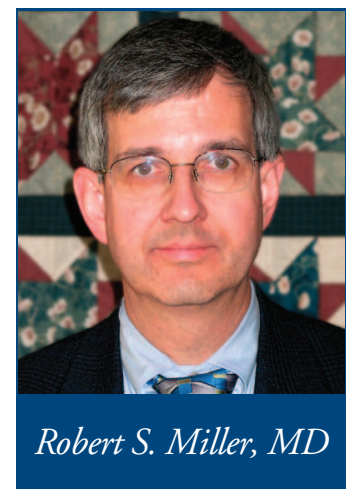

Editor's Note: IT (Information

Technology) Help Desk is a new

feature which will run from timeto-time in the JOP. We anticipate addressing, in a Q o A format, issues that may arise as oncologists implement new IT tools into their practices. Readers may submit questions of general interest to jopsubmissions@asco.org.

\section{How prevalent is the use of $e$-mail and other forms of} electronic communication between physicians and patients?

Although numerous reports have shown that patients are very interested in exchanging e-mail with their physicians, its use today in medical settings is surprisingly rare. In a 2002 survey of a representative sample of the US population, only $6 \%$ of respondents had corresponded via e-mail with their physician in the preceding year. ${ }^{1}$

\section{What are the barriers that limit e-mail use between patients and providers?}

Physicians are often biased against using e-mail with patients, so they rarely encourage it. They fear they will be overwhelmed with messages, and no mechanism currently exists for reimbursement for the time spent reading and composing e-mails. Physicians are also concerned about the medicolegal implications of urgent or sensitive content in patient e-mails, about preserving patient confidentiality, and about compliance with the Health Insurance Portability and Accountability Act (HIPAA).

\section{What are the advantages and disadvantages of e-mail in the clinical setting?}

E-mail is an asynchronous form of communication, well-suited to clinical practice. Its use can improve office efficiency by minimizing missed phone calls and voice messages. It provides a superior level of documentation compared with the manner in which most phone calls are documented. It is spontaneous and informal, and some patients may find it a less intimidating way of communicating with their physicians. Furthermore, it is widely available and inexpensive.

One disadvantage is that it is impersonal and not well-suited to some clinical topics. Furthermore, it is not time-sensitive, so it is not appropriate for urgent issues. It inherently lacks security; an e-mail message can be intercepted by someone with only a moderate level of technological sophistication, plus it is all too easy to misaddress or otherwise misdirect a message. Additionally, e-mail is unstructured and difficult to triage into an office workflow.

\section{What are the HIPAA implications of patient- physician e-mail?}

HIPAA does not expressly prohibit e-mail between patients and physicians, but e-mail with patients almost always contains protected health information (PHI) and must be safeguarded. While the HIPAA Security Rule does not require the formal digital encryption of e-mail, a practice not utilizing some form of security must justify its choices. Therefore, in most settings, the use of conventional, unencrypted e-mail would not be considered HIPAA-compliant.

\section{Are there other alternatives to traditional e-mail?}

Conventional e-mail can be encrypted using special software, but this can be cumbersome and may be a barrier to use of e-mail. A more attractive option is the use of Web-based messaging portals. These are secure, HIPAA-compliant Web sites, typically implemented by third party vendors, with a monthly fee charged per physician in a practice. They require each user to have a unique login and password, comparable to banking and travel sites. In addition to messaging capabilities, they may feature independent educational health content or built-in functionality such as electronic prescribing. Some are integrated into electronic health record (EHR) systems.

\section{What does the available clinical research on the use of electronic communication between patients and physicians show?}

Most studies have been done in primary care populations, commonly in large, multisite integrated-provider networks. Patients are generally very satisfied with e-mail and Webbased messaging, and often prefer it to the telephone for routine matters such as scheduling appointments or requesting prescription refills. Physicians find it a useful modality for the management of chronic diseases in patients they know well. ${ }^{2}$ Contrary to their initial biases, most physicians are not overwhelmed with messages from patients, and most patients are able to follow specific guidelines regarding its use. However, the data are mixed as to whether it truly saves time for the physician or decreases the volume of phone calls coming into an office. Currently, physician reimbursement for its use is meager to nonexistent, though some third-party payers are implementing programs to pay physicians for care rendered online.

\section{What guidelines should be followed by practices wishing to initiate electronic communication with patients?}

A number of organizations, such as the American Medical Informatics Association, have published pertinent guidelines. Examples include:

- Patients should be required to give informed consent so that they understand the appropriate use and limitations of electronic communication 
- Patients should be informed as to which members of the office staff will be handling the messages in addition to the physician

- Patients should be encouraged to be concise and to use transaction categories in the subject line of e-mails (eg, "Prescription Refill Request")

- Patients should be informed as to what clinical topics are inappropriate

- Turnaround time expectations for messages should be clearly established

- Auto-reply mechanisms should be configured to generate an automatic acknowledgement of the receipt of a patient message/request; a message should also be sent to confirm completion of the patient's request

- Offices should determine site-specific policies for triage of incoming messages; backup and retrieval mechanisms should be put into place (ie, it is not appropriate for patient e-mails simply to sit in the physician inbox)

- Sites that are not EHR-enabled should print the e-mail string and place in the patient's medical record

- E-mail messages should be written in plain text, not HTML, and attachments, if used, should be in PDF not Microsoft Word format

\section{Reference}

1. Baker $L$, Wagner $T$, Singer $S$, et al: Use of the Internet and e-mail for health care information: Results from a national survey. JAMA 289:2400-2406, 2003
- When appropriate, physician e-mails should include relevant URLs to reputable Web sites for patient education

- Physicians should be vigilant about "low-tech" risks to security: using an inadequate or static password, using a home e-mail account for patient messages, relying on auto-completion addressing by the e-mail client or accidentally hitting "Reply to All" instead of "Reply," and so forth

- If an e-mail/message string becomes too lengthy, it should be terminated and the patient scheduled for an office visit

\section{What does the future hold for the use of electronic communication between patients and physicians?}

It is here to stay and should be embraced by physicians, not avoided. Some patients are already basing their choice of physician on a demonstrated willingness to engage in e-mail exchange. Using electronic communication with patients can improve patient satisfaction and the quality of care, and it may enable physicians to make their office practices more efficient.

2. Liederman J, Lee J, Bacquero V, et al: Patient-physician web messaging: The impact on messaging volume and satisfaction. J Gen Intern Med 20:52-57, 2005

\section{Experience the 2006 ASCO Annual Meeting at Your Convenience}

ASCO.org now offers cutting-edge science from the Annual Meeting in a variety of convenient formats.

- WATCH presentations from the commemorative 3-DVD set or online via the Virtual Meeting

- LISTEN to sessions through the Audio Podcast or order tapes and CDs

- READ key publications including the Proceedings and the Educational Book

Your schedule. Your format. Your choice.

For more information, visit www.asco.org/annualmeeting 